\title{
Dental Health Behaviors, Dentition, and Mortality in the Elderly: The Leisure World Cohort Study
}

\author{
Annlia Paganini-Hill,, ${ }^{1,2}$ Stuart C. White, ${ }^{3}$ and Kathryn A. Atchison ${ }^{4}$ \\ ${ }^{1}$ Department of Preventive Medicine, Keck School of Medicine, University of Southern California, Los Angeles, CA 90033, USA \\ ${ }^{2}$ Department of Neurology, University of California, Irvine, CA 92697, USA \\ ${ }^{3}$ Oral and Maxillofacial Radiology Section, UCLA School of Dentistry, Los Angeles, CA 90095, USA \\ ${ }^{4}$ Public Health and Community Dentistry Divison, UCLA School of Dentistry, Los Angeles, CA 90095, USA \\ Correspondence should be addressed to Annlia Paganini-Hill, annliahi@usc.edu
}

Received 12 March 2011; Accepted 8 April 2011

Academic Editor: Bo A. Hagberg

Copyright ( 2011 Annlia Paganini-Hill et al. This is an open access article distributed under the Creative Commons Attribution License, which permits unrestricted use, distribution, and reproduction in any medium, provided the original work is properly cited.

\begin{abstract}
In the last decade the effect of oral health on the general health and mortality of elderly people has attracted attention. We explored the association of dental health behaviors and dentition on all-cause mortality in 5611 older adults followed from 1992 to 2009 (median $=9$ years) and calculated risk estimates using Cox regression analysis in men and women separately. Toothbrushing at night before bed, using dental floss everyday, and visiting the dentist were significant risk factors for longevity. Never brushing at night increased risk 20-35\% compared with brushing everyday. Never flossing increased risk 30\% compared with flossing everyday. Not seeing a dentist within the last 12 months increased risk 30-50\% compared with seeing a dentist two or more times. Mortality also increased with increasing number of missing teeth. Edentulous individuals (even with dentures) had a $30 \%$ higher risk of death compared with those with $20+$ teeth. Oral health behaviors help maintain natural, healthy and functional teeth but also appear to promote survival in older adults.
\end{abstract}

\section{Introduction}

Only in the last decade has the possible effect of oral health on the general health and mortality of elderly people attracted much attention. An association between number of teeth and mortality has been reported in several studies [1-11]. As people age, many lose teeth. Tooth loss reduces masticatory capacity, which can influence food selection, nutritional status, and general health. Evidence is also increasing that oral infections play a role in the pathogenesis of some systemic diseases and may be especially debilitating in the old and frail $[12,13]$.

The focus of the present study was to examine the possible role not only of dentition status, especially the number of natural teeth and use of dentures, but also of dental health practices as predictors of mortality in elderly men and women. In addition, we wanted to determine if any observed effects would remain after adjusting for other health and lifestyle factors related to mortality. We hypoth- esized that more teeth and better dental health behaviors would be related to increased longevity. We report here the results in a large cohort (over 5000) of elderly (median age 81 years) men and women followed for 17 years.

\section{Materials and Methods}

The Leisure World Cohort Study was established in the early 1980s when 13,978 (8877 female and 5101 male) residents of a California retirement community (Leisure World Laguna Hills) completed a postal health survey. Residents were recruited in four waves: those who owned homes in Leisure World on June 1, 1981; new residents who had moved into the community and were living there on June 1, 1982; on June 1, 1983; on October 1, 1985. The population and the cohort are mostly Caucasian, well educated, upper-middle class, and elderly.

The baseline survey asked demographic information (birth date, sex, marital status, number of children, height, and weight), brief medical history (high blood pressure, 
angina, heart attack, stroke, diabetes, rheumatoid arthritis, fractures after age 40, cancer, gallbladder surgery, glaucoma, and cataract surgery), medication use (hypertensive medication, digitalis, and nonprescription pain medication), personal habits (cigarette smoking, exercise, alcohol consumption, and vitamin supplement use), and beverage intake (milk, regular coffee, decaffeinated coffee, black or green tea, and soft drinks).

2.1. Dental Health Status and Behaviors. We mailed a follow-up survey to the 8403 cohort members still alive on November 1, 1992. Emphasis was placed on questions regarding dental health, focusing on the number of natural teeth, dentures worn, number of visits to a dentist, and oral health habits. Participants were asked to indicate how often they did the following: "brush my teeth in the morning, brush my teeth at night before bed, brush my teeth during the day, clean my dentures, use dental floss, use mouth wash, and use a tooth pick" with the response categories of everyday, sometimes, never. The survey was returned by 6173 cohort members (73\%). We were unable to contact 13 cohort members and another $384(5 \%)$ died within one year of the mailing. Of those returning the survey, 487 did not indicate the number of teeth or provide information on any dental health practice and were eliminated from the analyses.

Jackson and Murray suggested 16 teeth as the lowest acceptable number of natural teeth in persons older than 60 years for adequate masticatory function [14]. We used this categorization with the additional division of $16+$ teeth into 16-25 and 26+ teeth. This was further refined to 10 teeth in the upper jaw and six in the lower jaw [15]. We used this latter criteria to classify our subjects into two groups: those with or without adequate natural dentition. Other studies have defined adequate dental status as at least 20 teeth [2], and we used this as an alternative definition, further classifying those with fewer than 20 teeth as wearing dentures or not.

2.2. Lifestyle Factors. Information on the lifestyle factors came from the original health survey completed in the early 1980s. We previously reported the effects of several lifestyle practices on all-cause mortality in this cohort [16-20]. Other studies have shown that body mass index and obesity [21, $22]$, smoking [23-25], alcohol consumption [25, 26], and coffee intake [26] are related to the number of teeth.

Based on their reported smoking history we classified participants as never, past, or current smokers [16].

Consumption of alcoholic beverages was asked separately for wine $(4 \mathrm{oz})$, beer $(12 \mathrm{oz})$, and hard liquor $(1 \mathrm{oz})$, each equivalent to about $1 / 2 \mathrm{oz}$ of alcohol. Response choices for average weekday consumption were never drink, less than 1 , $1,2,3$, and 4 or more drinks. Total alcohol intake per day was calculated by summing the number of drinks consumed of each type [17]. Individuals were then categorized into four groups: $0, \leq 1,2-3$, and $4+$ drinks/day.

We estimated daily caffeine intake by summing the frequency of consumption of each beverage and chocolate multiplied by its average caffeine content ( $\mathrm{mg} /$ standard unit) as $115,3,50,50$, and 6 for regular coffee, decaffeinated coffee, tea, cola soft drinks, and chocolate, respectively [18]. Caffeine intake was categorized as $<50,50-99,100-199,200-$ $399,400+\mathrm{mg} /$ day.

The amount of time spent on physical activities was ascertained by asking the amount of time spent in active and other (less physically demanding) activities. Activities were categorized as $0, \leq 1 / 2,3 / 4-1,2+$ hours/day for active activities and $<2,2-3,4-5,6+$ hours/day for other activities [19].

Body mass index (weight $(\mathrm{kg}) /$ height $(\mathrm{m})^{2}$ ) was calculated based on self-reported height and weight at baseline and categorized according to federal guidelines: underweight (<18.5), normal weight (18.5-24.9), overweight (25-29.9), and obese $(30+)[20,27]$.

2.3. Determination of Outcome. Followup of the cohort is maintained by periodic resurvey and determination of vital status by search of governmental and commercial death indexes and ascertainment of death certificates. Participants were followed to death or December 31, 2009, whichever came first. To date, 40 cohort members have been lost to followup; search of death indices did not reveal that these individuals were deceased.

2.4. Statistical Analysis. Hazard ratios (HRs) and $95 \%$ confidence intervals (CIs) were obtained using Cox proportional hazard regression analysis [28]. For the Cox models, chronological age was used as the fundamental time scale with study entry being age in 1992 when the dental health survey was completed and the event of interest being age at death. Because women differ from men on many of the dental and potential confounding variables, women live longer on average than men, and for comparison with other studies which stratified by sex, we performed separate analyses for men and women. HRs were calculated for dental status and each dental health behavior adjusted for age (continuous) and then additionally adjusted for lifestyle variables plus seven separate histories (no, yes) of hypertension, angina, heart attack, stroke, diabetes, rheumatoid arthritis, and cancer, previously found to be significant risk factors for death in this cohort [16-20]. For some dental health behaviors separate analyses were done for subjects with teeth and for subjects with dentures. Statistical analyses were performed using SAS version 9.2 (SAS Institute Inc., Cary, NC). No adjustment in the $P$ values was made for multiple comparisons.

Previous reports present details of the methods and validity of exposure and outcome data [16-20, 29-31]. The Institutional Review Boards of the University of Southern California and the University of California, Los Angeles approved the study.

\section{Results}

After excluding 65 subjects with missing covariates, we analyzed data on 5611 subjects ( 3847 women and $1764 \mathrm{men}$ ). At study entry, the participants ranged in age from 52 to 105 years (median, 81 years). By December 31, 2009, the subjects had contributed 52,441 person-years of followup (median 9 
TABLE 1: Characteristics of the cohort by sex.

\begin{tabular}{|c|c|c|c|c|}
\hline \multirow{3}{*}{ Number } & \multirow{2}{*}{\multicolumn{2}{|c|}{$\begin{array}{l}\text { Men } \\
1764\end{array}$}} & \multirow{2}{*}{\multicolumn{2}{|c|}{$\begin{array}{c}\text { Women } \\
3847\end{array}$}} \\
\hline & & & & \\
\hline & Mean & SD & Mean & $\mathrm{SD}$ \\
\hline Age at time of dental survey (years) & 81 & 6.5 & 80 & 7.1 \\
\hline Age at last followup (years) & 89 & 5.9 & 90 & 6.2 \\
\hline Followup years & 8.2 & 5.2 & 9.9 & 5.3 \\
\hline \multicolumn{5}{|l|}{ At time of original survey (1981-1985): } \\
\hline Active activities (hrs/day) & 1.2 & 1.3 & 1.1 & 1.2 \\
\hline Other activities (hrs/day) & 3.9 & 2.8 & 4.8 & 2.7 \\
\hline Alcohol (drinks/day) & 1.7 & 1.5 & 1.3 & 1.2 \\
\hline Caffeine (mg/day) & 190 & 176 & 182 & 169 \\
\hline Body mass index $\left(\mathrm{kg} / \mathrm{m}^{2}\right)$ & 25 & 2.7 & 24 & 3.5 \\
\hline \multirow[t]{2}{*}{ Number of natural teeth } & 19.0 & 10.5 & 20.5 & 9.9 \\
\hline & No. & $\%$ & No. & $\%$ \\
\hline \multicolumn{5}{|l|}{ Medical history } \\
\hline High blood pressure & 572 & 32 & 1367 & 36 \\
\hline Angina & 192 & 11 & 275 & 7.1 \\
\hline Heart attack & 212 & 12 & 154 & 4.0 \\
\hline Stroke & 58 & 3.3 & 63 & 1.6 \\
\hline Cancer & 99 & 5.6 & 444 & 12 \\
\hline Diabetes & 101 & 5.7 & 134 & 3.5 \\
\hline Rheumatoid arthritis & 58 & 3.3 & 216 & 5.6 \\
\hline \multicolumn{5}{|l|}{ Smoke } \\
\hline Never & 651 & 37 & 2065 & 54 \\
\hline Past & 980 & 56 & 1320 & 34 \\
\hline Current & 133 & 7 & 462 & 12 \\
\hline Dentures & 924 & 52 & 1703 & 44 \\
\hline Deceased by December 31, 2009 & 1588 & 90 & 3165 & 82 \\
\hline
\end{tabular}

years), and 4753 had died. Age at death ranged from 64 to 108 years (median, 90 years).

Table 1 presents selected characteristics for the participants by sex. Differences between males and females were highly statistically significant $(P<.01)$ for all variables except age at study entry $(P=.32)$ and caffeine $(P=.11)$. The median number of teeth in cohort members was 24 (range $0-32$, mean 20) and nearly half had dentures. Men tended to have fewer teeth than women (median 23 versus 24 ) and had a greater frequency of denture use. Only $11 \%$ of the cohort was edentulous.

Table 2 shows the age-adjusted and mutivariable-adjusted HRs of all-cause mortality for number of teeth and the various definitions of adequate dentition for women and men, separately. Adjustment for potential confounders had limited effect, generally attenuating the observed HRs. Number of natural teeth was related to longevity in both men and women. Edentulous individuals had a $30 \%$ higher risk of death than subjects with 26-32 teeth; those with 1-15 teeth had a $20 \%$ higher risk. Compared with those with adequate natural masticatory function (10+ upper teeth and $6+$ lower teeth), those with inadequate function had over a $15 \%$ in- creased risk of death. Ninety percent of subjects with inadequate natural masticatory function had dentures. Among women with inadequate natural masticatory function, those without dentures had a somewhat higher (not significant) risk of death than those with dentures. No difference was seen in men.

Table 3 shows the HRs for the dental health behaviors in individuals with teeth. Daily tooth brushing at night before bed and flossing significantly decreased risk of death while using a tooth pick or mouth wash did not. Both individuals who never brushed at night and those who never flossed had a $20-30 \%$ increased mortality risk compared to those who brushed at night or flossed everyday, respectively. Those who reported not brushing their teeth daily had a 41-91\% increased risk of death compared with those who brushed three times daily-in the morning, during the day and at night. Brushing at night was the most significant tooth brushing variable. Risk was similar for those brushing at night everyday whether or not they brushed daily in the morning or during the day. Individuals who everyday brushed in the morning or during the day but not everyday at night had a $13-26 \%$ increased risk. 
TABLE 2: Hazard ratios for mortality by dental status, Leisure World Cohort Study, 1992-2009.

\begin{tabular}{|c|c|c|c|c|c|c|c|c|c|c|}
\hline & \multicolumn{5}{|c|}{ Men } & \multicolumn{5}{|c|}{ Women } \\
\hline & \multirow{2}{*}{ No. } & \multicolumn{2}{|c|}{ Model 1* } & \multicolumn{2}{|c|}{ Model 2* } & \multirow{2}{*}{ No. } & \multicolumn{2}{|c|}{ Model 1* } & \multicolumn{2}{|c|}{ Model 2* } \\
\hline & & HR & $95 \% \mathrm{CI}$ & HR & $95 \% \mathrm{CI}$ & & $\mathrm{HR}$ & $95 \% \mathrm{CI}$ & HR & $95 \% \mathrm{CI}$ \\
\hline \multicolumn{11}{|c|}{ Number of natural teeth } \\
\hline $26-32$ & 659 & 1.00 & & 1.00 & & 1709 & 1.00 & & 1.00 & \\
\hline $16-25$ & 541 & 1.04 & $0.92-1.18$ & 1.03 & $0.91-1.17$ & 1131 & 1.01 & $0.93-1.10$ & 0.97 & $0.89-1.06$ \\
\hline $1-15$ & 343 & 1.22 & $1.07-1.41$ & 1.21 & $1.05-1.40$ & 627 & 1.23 & $1.11-1.36$ & 1.17 & $1.06-1.30$ \\
\hline 0 & 221 & 1.33 & $1.13-1.56$ & 1.18 & $1.00-1.39$ & 380 & 1.30 & $1.15-1.47$ & 1.21 & $1.07-1.37$ \\
\hline \multicolumn{11}{|c|}{ Number of natural teeth } \\
\hline $20-32$ & 1053 & 1.00 & & 1.00 & & 2551 & 1.00 & & 1.00 & \\
\hline $1-19$ & 490 & 1.11 & $0.99-1.25$ & 1.09 & $0.97-1.23$ & 916 & 1.19 & $1.10-1.30$ & 1.15 & $1.06-1.25$ \\
\hline $\begin{array}{l}\text { (i) Dentures- } \\
\text { yes }\end{array}$ & 446 & 1.12 & $1.00-1.30$ & 1.10 & $0.98-1.24$ & 809 & 1.19 & $1.09-1.30$ & 1.15 & $1.05-1.25$ \\
\hline $\begin{array}{l}\text { (ii) Dentures- } \\
\text { no }\end{array}$ & 44 & 1.03 & $0.75-1.40$ & 1.01 & $0.74-1.39$ & 107 & 1.21 & $0.99-1.49$ & 1.16 & $0.95-1.43$ \\
\hline 0 & 221 & 1.29 & $1.11-1.51$ & 1.15 & $0.98-1.34$ & 380 & 1.31 & $1.16-1.47$ & 1.24 & $1.10-1.39$ \\
\hline \multicolumn{11}{|c|}{ Adequate masticatory function: $10+$ upper teeth and $6+$ lower teeth } \\
\hline Yes & 1017 & 1.00 & & 1.00 & & 2498 & 1.00 & & 1.00 & \\
\hline No & 747 & 1.16 & $1.05-1.29$ & 1.12 & $1.01-1.24$ & 1349 & 1.22 & $1.13-1.31$ & 1.17 & $1.08-1.26$ \\
\hline $\begin{array}{l}\text { (i) Dentures- } \\
\text { yes }\end{array}$ & 680 & 1.16 & $1.05-1.29$ & 1.11 & $1.00-1.24$ & 1205 & 1.21 & $1.12-1.30$ & 1.16 & $1.07-1.25$ \\
\hline $\begin{array}{l}\text { (ii) Dentures- } \\
\text { no }\end{array}$ & 67 & 1.18 & $0.91-1.52$ & 1.19 & $0.92-1.55$ & 144 & 1.33 & $1.12-1.59$ & 1.25 & $1.05-1.50$ \\
\hline \multicolumn{11}{|c|}{ Dentures } \\
\hline None & 766 & 1.00 & & 1.00 & & 1786 & 1.00 & & 1.00 & \\
\hline $\begin{array}{l}\text { Partial or partial } \\
\text { plus one jaw full }\end{array}$ & 711 & 1.08 & $0.97-1.21$ & 1.06 & $0.95-1.18$ & 1359 & 1.08 & $1.00-1.17$ & 1.05 & $0.97-1.14$ \\
\hline $\begin{array}{l}\text { Full upper and } \\
\text { lower }\end{array}$ & 213 & 1.29 & $1.10-1.51$ & 1.14 & $0.96-1.34$ & 344 & 1.22 & $1.08-1.38$ & 1.14 & $1.01-1.30$ \\
\hline Unknown & 74 & 1.25 & $0.97-1.60$ & 1.27 & $0.99-1.64$ & 358 & 0.94 & $0.83-1.07$ & 0.92 & $0.81-1.04$ \\
\hline
\end{tabular}

* Model 1: adjusted for age at entry.

Model 2: adjusted for age at entry, smoking, alcohol, caffeine, active activities, other activities, body mass index, high blood pressure, angina, heart attack, stroke, diabetes, rheumatoid arthritis, and cancer.

To sort out whether brushing teeth at night or flossing was more important we looked at both together. Among subjects who brushed their teeth at night everyday, never flossing conferred a significantly increased risk of death of about $25 \%$ compared with those who flossed everyday. Among subjects who used dental floss everyday, those never brushing their teeth at night had similarly higher risk than subjects who brushed everyday, but the risk was not statistically significant. However, the numbers never brushing were small, 33 men and 63 women, and the estimates of risk were similar to that of the all subjects who never brushed at night. Never brushing teeth at night and never flossing remained significant risk factors after adjusting for adequate dentition. The risk of not brushing was 1.29 (95\% CI 1.10-1.51) in men and 1.18 (95\% CI 1.01-1.36) in women. The risk of not flossing was 1.25 (95\% CI 1.08-1.43) in men and 1.29 (95\% CI 1.16-1.42) in women.

Table 4 shows the HRs for the dental health behaviors in individuals with dentures. Never cleaning dentures was a significant risk factor in men $(\mathrm{HR}=1.25)$ but not in women. Use of mouthwash had no effect on mortality.

Compared with having visited a dentist twice in the last 12 months, not seeing a dentist was associated with a higher $(25-50 \%)$ risk of death in both men and women and in both those with teeth and those with dentures. The risk was slightly higher in men than women and in those with teeth than in those with dentures.

\section{Discussion}

Our study extends the available literature on the survival benefits of adequate dentition, use of dentures among those without adequate natural masticatory function, brushing teeth at night before bed, and flossing in the elderly. Each of these was associated with reduced death in our elderly men and women. Although brushing teeth at night before bed and flossing were correlated $(r=0.23)$, each independently predicted risk. 
TABLE 3: Hazard ratios for mortality by dental health behaviors among subjects with teeth, Leisure World Cohort Study, $1992-2009$.

\begin{tabular}{|c|c|c|c|c|c|c|c|c|c|c|}
\hline & \multicolumn{5}{|c|}{ Men } & \multicolumn{5}{|c|}{ Women } \\
\hline & \multirow{2}{*}{ No. } & \multicolumn{2}{|c|}{ Model $1^{*}$} & \multicolumn{2}{|c|}{ Model 2* } & \multirow{2}{*}{ No. } & \multicolumn{2}{|c|}{ Model 1* } & \multicolumn{2}{|c|}{ Model 2* } \\
\hline & & HR & $95 \% \mathrm{CI}$ & HR & $95 \% \mathrm{CI}$ & & HR & $95 \% \mathrm{CI}$ & HR & $95 \% \mathrm{CI}$ \\
\hline \multicolumn{11}{|c|}{ Brush my teeth in the morning } \\
\hline Everyday & 1196 & 1.00 & & 1.00 & & 3014 & 1.00 & & 1.00 & \\
\hline Sometimes & 176 & 1.03 & $0.87-1.21$ & 1.08 & $0.91-1.29$ & 250 & 1.14 & $0.99-1.31$ & 1.13 & $0.98-1.30$ \\
\hline Never & 171 & 0.94 & $0.79-1.12$ & 0.95 & $0.80-1.12$ & 203 & 0.99 & $0.85-1.15$ & 151.01 & $0.86-1.17$ \\
\hline \multicolumn{11}{|c|}{ Brush my teeth at night before bed } \\
\hline Everyday & 1112 & 1.00 & & 1.00 & & 2895 & 1.00 & & 1.00 & \\
\hline Sometimes & 222 & 1.17 & $1.01-1.37$ & 1.13 & $0.97-1.32$ & 350 & 1.17 & $1.03-1.32$ & 1.13 & $1.00-1.28$ \\
\hline Never & 209 & 1.36 & $1.16-1.58$ & 1.34 & $1.14-1.57$ & 222 & 1.19 & $1.02-1.38$ & 1.19 & $1.02-1.38$ \\
\hline \multicolumn{11}{|c|}{ Brush my teeth during the day } \\
\hline Everyday & 265 & 1.00 & & 1.00 & & 965 & 1.00 & & 1.00 & \\
\hline Sometimes & 521 & 0.99 & $0.85-1.16$ & 0.95 & $0.81-1.12$ & 1498 & 1.01 & $0.92-1.10$ & 0.98 & $0.90-1.07$ \\
\hline Never & 757 & 1.09 & $0.94-1.26$ & 1.06 & $0.91-1.23$ & 1004 & 1.14 & $1.03-1.25$ & 1.10 & $1.00-1.21$ \\
\hline \multicolumn{11}{|c|}{ Use dental floss } \\
\hline Everyday & 462 & 1.00 & & 1.00 & & 1572 & 1.00 & & 1.00 & \\
\hline Sometimes & 603 & 1.18 & $1.03-1.34$ & 1.14 & $1.00-1.30$ & 1226 & 1.05 & $0.97-1.14$ & 1.03 & $0.95-1.12$ \\
\hline Never & 478 & 1.31 & $1.15-1.50$ & 1.27 & $1.11-1.46$ & 669 & 1.31 & $1.19-1.45$ & 1.28 & $1.16-1.42$ \\
\hline \multicolumn{11}{|c|}{ Use mouth wash } \\
\hline Everyday & 400 & 1.00 & & 1.00 & & 1134 & 1.00 & & 1.00 & \\
\hline Sometimes & 543 & 0.99 & $0.87-1.14$ & 1.01 & $0.88-1.16$ & 1249 & 1.00 & $0.91-1.09$ & 0.98 & $0.90-1.07$ \\
\hline Never & 600 & 0.92 & $0.80-1.05$ & 0.93 & $0.81-1.07$ & 1084 & 0.98 & $0.89-1.07$ & 1.00 & $0.91-1.10$ \\
\hline \multicolumn{11}{|c|}{ Use a tooth pick } \\
\hline Everyday & 363 & 1.00 & & 1.00 & & 710 & 1.00 & & 1.00 & \\
\hline Sometimes & 661 & 0.93 & $0.81-1.07$ & 0.95 & $0.83-1.10$ & 1306 & 0.99 & $0.89-1.10$ & 0.98 & $0.88-1.09$ \\
\hline Never & 519 & 1.00 & $0.87-1.15$ & 1.01 & $0.88-1.16$ & 1451 & 1.05 & $0.95-1.16$ & 1.04 & $0.94-1.15$ \\
\hline \multicolumn{11}{|c|}{ Brush my teeth } \\
\hline $\begin{array}{l}\text { Everyday } \\
\text { morning, day } \\
\text { and night }\end{array}$ & 217 & 1.00 & & 1.00 & & 870 & 1.00 & & 1.00 & \\
\hline $\begin{array}{l}\text { Everyday } \\
\text { morning and } \\
\text { night, not } \\
\text { everyday day }\end{array}$ & 646 & 1.06 & $0.90-1.24$ & 1.01 & $0.86-1.20$ & 1679 & 1.04 & $0.95-1.14$ & 1.01 & $0.93-1.11$ \\
\hline $\begin{array}{l}\text { Everyday } \\
\text { night, not } \\
\text { everyday } \\
\text { morning/day }\end{array}$ & 249 & 0.96 & $0.80-1.17$ & 0.96 & $0.79-1.17$ & 346 & 1.00 & $0.87-1.14$ & 0.98 & $0.86-1.13$ \\
\hline $\begin{array}{l}\text { Everyday } \\
\text { morning/day, } \\
\text { not everyday } \\
\text { night }\end{array}$ & 350 & 1.26 & $1.06-1.51$ & 1.19 & $0.99-1.43$ & 496 & 1.13 & $1.00-1.28$ & 1.09 & $0.97-1.24$ \\
\hline Not everyday & 81 & 1.41 & $1.08-1.83$ & 1.37 & $1.05-1.80$ & 76 & 1.91 & $1.49-2.44$ & 1.77 & $1.38-2.28$ \\
\hline \multicolumn{11}{|c|}{ Brush my teeth at night before bed among subjects who use dental floss everyday } \\
\hline Everyday & 403 & 1.00 & & 1.00 & & 1432 & 1.00 & & 1.00 & \\
\hline Sometimes & 26 & 1.20 & $0.79-1.82$ & 1.28 & $0.82-1.99$ & 77 & 0.94 & $0.72-1.23$ & 0.87 & $0.66-1.15$ \\
\hline Never & 33 & 1.36 & $0.92-1.99$ & 1.33 & $0.87-2.01$ & 63 & 1.23 & $0.93-1.62$ & 1.21 & $0.91-1.60$ \\
\hline \multicolumn{11}{|c|}{ Use dental floss among subjects who brush teeth at night before bed everyday } \\
\hline Everyday & 403 & 1.00 & & 1.00 & & 1432 & 1.00 & & 1.00 & \\
\hline Sometimes & 422 & 1.17 & $1.01-1.36$ & 1.14 & $0.98-1.33$ & 988 & 1.04 & $0.95-1.14$ & 1.02 & $0.93-1.12$ \\
\hline Never & 287 & 1.26 & $1.08-1.48$ & 1.25 & $1.06-1.48$ & 475 & 1.24 & $1.11-1.39$ & 1.20 & $1.07-1.34$ \\
\hline
\end{tabular}


TABLe 3: Continued.

\begin{tabular}{|c|c|c|c|c|c|c|c|c|c|c|}
\hline & \multicolumn{5}{|c|}{ Men } & \multicolumn{5}{|c|}{ Women } \\
\hline & \multirow{2}{*}{ No. } & \multicolumn{2}{|c|}{ Model $1^{*}$} & \multicolumn{2}{|c|}{ Model 2* } & \multirow{2}{*}{ No. } & \multicolumn{2}{|c|}{ Model $1 *$} & \multicolumn{2}{|c|}{ Model 2* } \\
\hline & & HR & $95 \% \mathrm{CI}$ & HR & $95 \% \mathrm{CI}$ & & HR & $95 \% \mathrm{CI}$ & HR & $95 \% \mathrm{CI}$ \\
\hline \multicolumn{11}{|c|}{ Number of dental visits within the last 12 months } \\
\hline $2+$ & 1173 & 1.00 & & 1.00 & & 2671 & 1.00 & & 1.00 & \\
\hline 1 & 235 & 1.05 & $0.91-1.22$ & 1.06 & $0.91-1.23$ & 505 & 1.04 & $0.94-1.16$ & 1.07 & $0.96-1.18$ \\
\hline 0 & 135 & 1.48 & $1.23-1.79$ & 1.46 & $1.21-1.77$ & 291 & 1.33 & $1.17-1.51$ & 1.31 & $1.14-1.50$ \\
\hline
\end{tabular}

* Model 1: adjusted for age at entry.

Model 2: adjusted for age at entry, smoking, alcohol, caffeine, active activities, other activities, body mass index, high blood pressure, angina, heart attack, stroke, diabetes, rheumatoid arthritis, and cancer.

TABLE 4: Hazard ratios for mortality by dental health behaviors among subjects with dentures, Leisure World Cohort Study, $1992-2009$.

\begin{tabular}{|c|c|c|c|c|c|c|c|c|c|c|}
\hline & \multicolumn{5}{|c|}{ Men } & \multicolumn{5}{|c|}{ Women } \\
\hline & \multirow{2}{*}{ No. } & \multicolumn{2}{|c|}{ Model 1* } & \multicolumn{2}{|c|}{ Model 2* } & \multirow{2}{*}{ No. } & \multicolumn{2}{|c|}{ Model $1^{*}$} & \multicolumn{2}{|c|}{ Model 2* } \\
\hline & & HR & $95 \% \mathrm{CI}$ & HR & $95 \% \mathrm{CI}$ & & HR & $95 \% \mathrm{CI}$ & HR & $95 \% \mathrm{CI}$ \\
\hline \multicolumn{11}{|c|}{ Clean my dentures } \\
\hline Everyday & 743 & 1.00 & & 1.00 & & 1443 & 1.00 & & 1.00 & \\
\hline Sometimes & 90 & 1.11 & $0.88-1.39$ & 1.11 & $0.87-1.40$ & 87 & 1.13 & $0.91-1.42$ & 1.09 & $0.87-1.38$ \\
\hline Never & 165 & 1.23 & $1.03-1.46$ & 1.24 & $1.03-1.48$ & 531 & 0.85 & $0.76-0.94$ & 0.87 & $0.78-0.97$ \\
\hline \multicolumn{11}{|c|}{ Use mouth wash } \\
\hline Everyday & 297 & 1.00 & & 1.00 & & 751 & 1.00 & & 1.00 & \\
\hline Sometimes & 308 & 0.91 & $0.77-1.08$ & 0.93 & $0.78-1.10$ & 668 & 0.95 & $0.85-1.07$ & 0.95 & $0.85-1.06$ \\
\hline Never & 393 & 0.99 & $0.84-1.15$ & 1.02 & $0.87-1.20$ & 642 & 0.96 & $0.86-1.08$ & 0.99 & $0.88-1.11$ \\
\hline \multicolumn{11}{|c|}{ Number of dental visits within the last 12 months } \\
\hline $2+$ & 612 & 1.00 & & 1.00 & & 1310 & 1.00 & & 1.00 & \\
\hline 1 & 137 & 1.04 & $0.86-1.26$ & 1.09 & $0.89-1.33$ & 299 & 1.09 & $0.95-1.25$ & 1.10 & $0.96-1.26$ \\
\hline 0 & 249 & 1.33 & $1.14-1.55$ & 1.23 & $1.05-1.45$ & 452 & 1.26 & $1.12-1.42$ & 1.20 & $1.07-1.35$ \\
\hline
\end{tabular}

${ }^{*}$ Model 1: adjusted for age at entry.

Model 2: adjusted for age at entry, smoking, alcohol, caffeine, active activities, other activities, body mass index, high blood pressure, angina, heart attack, stroke, diabetes, rheumatoid arthritis, and cancer.

In the present study, the associations between mortality and the number of teeth and the dental health behaviors were to a large extent independent of established risk factors for mortality. Although lifestyle factors such as smoking, alcohol, and caffeine consumption play important roles in relation to both survival and dental health, the HRs adjusted for these and other confounders were similar to those adjusted for age only. Conscientiousness and other personality traits undoubtedly play a role in health-related behaviors and longevity [32,33]. However, that only toothbrushing at night and flossing and not toothbrushing in the morning and using mouth wash were related to reduced risk of death suggests that these behaviors are directly related to longevity.

We acknowledge several limitations in our study. All variables used in the analyses are self-reported; we performed no dental examinations. However, previous studies in our population and others support the reliability of self-reported number of teeth [10], health practices and drug usage [29], medical history of major chronic disease $[29,30]$, and height and weight [29]. Another limitation is that changes over time in all potential risk factors may affect outcome. Additionally, the subjects in our study were mostly white, highly educated, and of middle-class social-economic status (SES), and therefore not representative of the general population. Although this may limit the generalizability of our results, it offers the advantage of reduced potential confounding by race, education, SES, and presumed access to health care. Tooth loss is typically the result of trauma, caries, or periodontal disease and is correlated with lower SES. Confounding by SES is unlikely to explain our results. Nonetheless, although we adjusted for other risk and potential confounding factors, unrecognized and uncontrolled confounders cannot be ruled out in this or any observational study.

This cohort has the advantages of population-based prospective design, large sample size, inclusion of men and women, and data on several potential confounders, including lifestyle practices and chronic disease history previously found to be related to mortality. The long and almost complete followup of the cohort resulted in a large number of outcome events.

Previous studies have identified dental factors that promote health and increase longevity (Table 5) [1-11]. Generally, the smaller the number of teeth the higher the risk of death. The robustness of the finding is illustrated by 


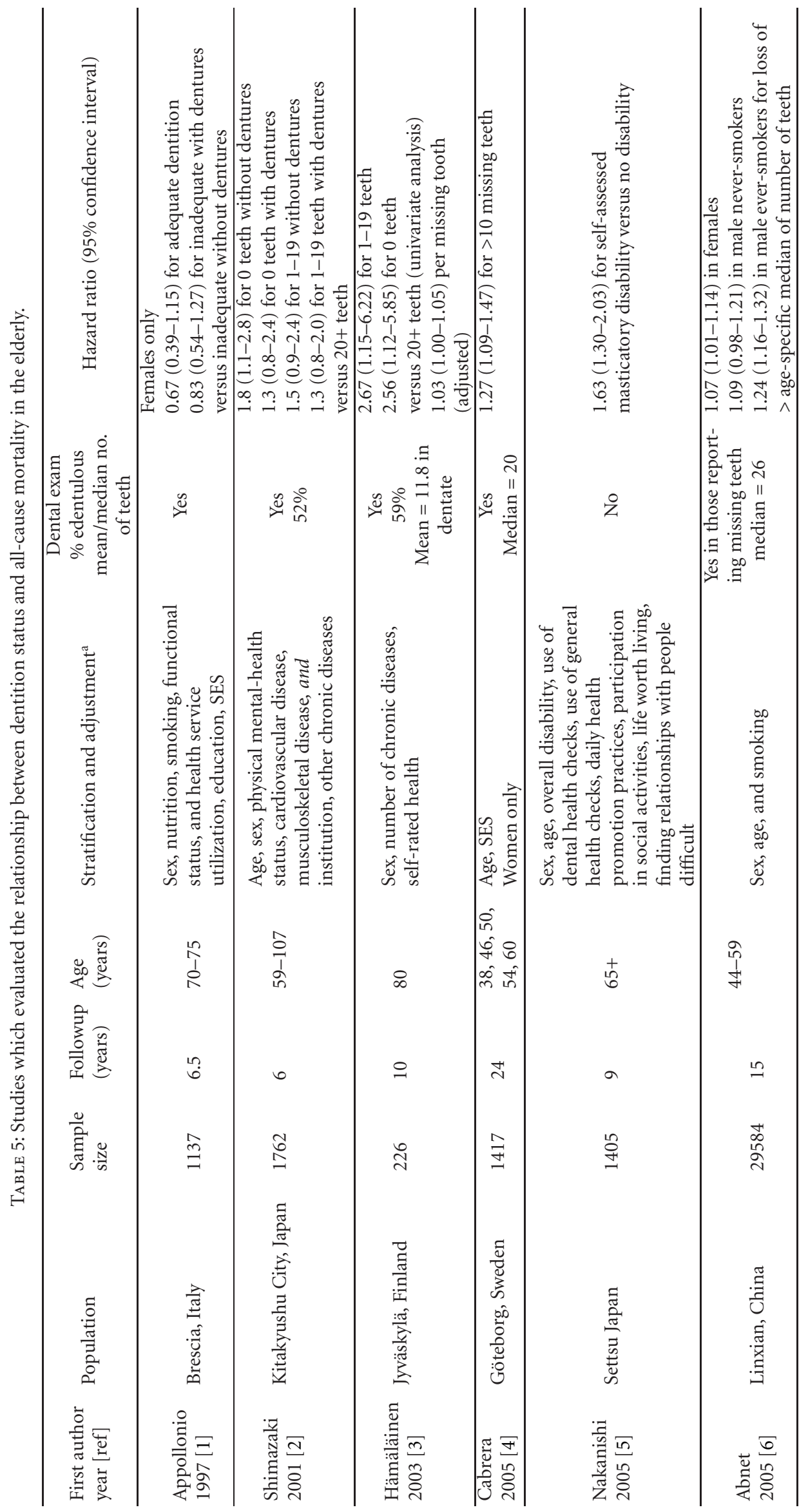




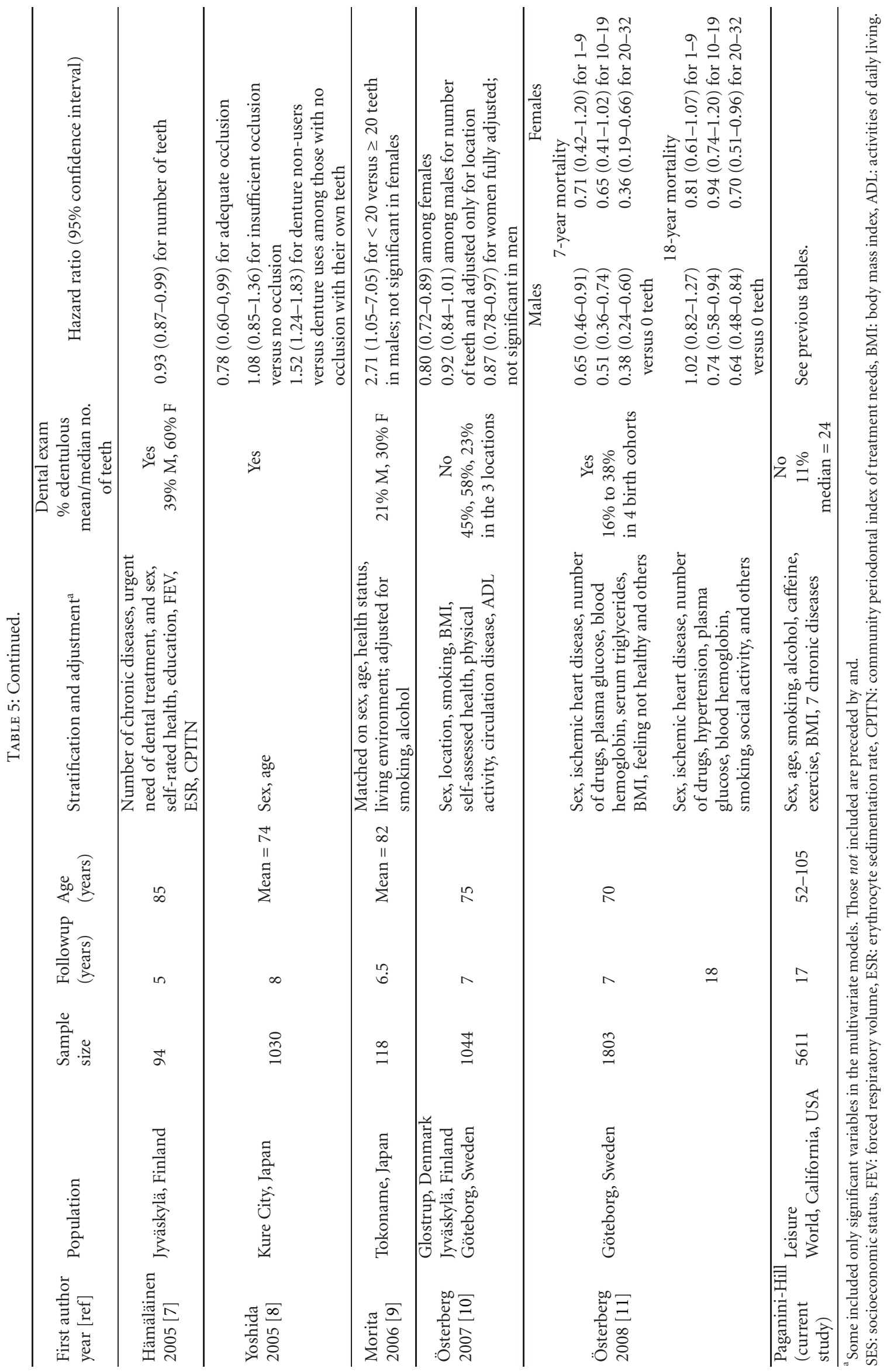


the various populations studied (Italy [1], Scandinavia [3, 4, 7, 10, 11], Japan [2, 5, 8, 9], China [6], and USA (present study)), the different classification schemes for number of teeth, the proportion of subjects edentulous (11 to 60\%), the mean/median number of teeth (11 to 26 ), the length of followup ( 5 to 24 years), the ages of the subjects (38 to 105 ), and the adjustments made for a wide variety of health and lifestyle founders that could be potential confounders. Some studies have found significant effects only in one sex group $[1,9,10]$. However, as in our study, loss of teeth is generally seen as a mortality risk factor in both men and women. The number of teeth appears to be a significant predictor of mortality independent of age, sex, education, SES, physical and mental health, body mass index, physical activity, smoking, alcohol consumption, caffeine intake, and country of origin.

Edentulous men and women in our study had a 30\% increased 17 -year mortality risk compared with those with $20+$ teeth. In Sweden, edentulous subjects had 2.8 (95\% CI 2.0-4.0) times greater 7-year mortality risk [11], similar to the Finnish study for 10-year mortality [3]. The 18-mortality risk in a Swedish study was lower at about $1.5(95 \% \mathrm{CI}=1.1-$ 2.8) times, similar to our finding of 1.3. This suggests that change over time in the risk factor may affect outcome.

Although the wearing of dentures may have a beneficial effect on mastication in those who have lost teeth, our study and others $[1,2]$ suggest that prostheses do not sufficiently compensate in terms of survival. Our study showed that edentulous subjects (virtually all who had prostheses) had an increase in mortality of 1.3 compared with those with 20 or more teeth. The corresponding ratio in Shimazaki et al. [2] was 1.3 (adjusted for physical-mental health status) and 1.2 compared with adequate natural dentition in Appollonio et al. [1]. Although in one Japanese study [8], the risk of mortality among denture non-users was 1.52-times (95\% $\mathrm{CI}=1.24-1.83$ ) higher than the risk for denture wearers in subjects who had no occlusal contact (with or without remaining teeth), the group as a whole had 1.3-times 8-year mortality rate of subjects who retained adequate occlusal contacts.

Most of the missing teeth of an elderly subject would have been infected before tooth loss or extractions, and, hence, the number of missing teeth would be a surrogate for previous dental infection. Poor dental status (periodontitis, dental bacteraemia, and oral mucosal infections) links with heart disease, stroke, atherosclerosis, pneumonia, other respiratory disease, and mortality (for a review see [12]). Oral infections may trigger systemic inflammatory reactions and death. Poorer masticatory ability and nutrient intake may also affect general health and survival.

Our study also found that several dental health behaviors were related to mortality. Not visiting a dentist within the last 12 months was significantly related to increased (30-50\% higher) mortality in both men and women and in both those with teeth and those with dentures. In a study in Settsu, Japan [5], not using dental health checks was also associated with mortality (HR $=1.4,95 \%$ CI $0.98-1.92)$.

Oral hygiene measures used in conjunction with regular professional care can prevent caries and most periodontal disease [34]. Toothbrushing and flossing are the most commonly used measures. Toothbrushing with a fluoride toothpaste helps prevent caries and dental floss is the most effective means of removing interdental plaque and reducing interdental gingival inflammations. Our study highlights the advantages on longevity of brushing at night and of flossing everyday.

Although not establishing a cause and effect relationship between mortality and dental status and behaviors, these strong and statistically persistent associations of dental health and mortality provide another basis for encouraging oral health and hygiene and use of dentures. One marker of successful ageing is the maintenance of a natural, healthy, and functional dentition. Retaining healthy teeth not only helps in mastication, nutrition, aesthetics, and self-esteem, but also appears to promote survival.

\section{Conclusion}

Results in this large elderly cohort with long followup showing a decreased risk of mortality with several dental health behaviors, number of teeth, and dentures suggest that maintenance of good oral hygiene is an important health promoter in aging populations.

\section{Acknowledgments}

This paper was funded by Grants from the National Institutes of Health (R01CA32197, R01AR47529, and R01AG21055), the Errol Carroll Trust Fund, and Wyeth-Ayerst Laboratories.

\section{References}

[1] I. Appollonio, C. Carabellese, A. Frattola, and M. Trabucchi, "Influence of dental status on dietary intake and survival in community-dwelling elderly subjects," Age and Ageing, vol. 26, no. 6, pp. 445-455, 1997.

[2] Y. Shimazaki, I. Soh, T. Saito et al., "Influence of dentition status on physical disability, mental impairment, and mortality in institutionalized elderly people," Journal of Dental Research, vol. 80, no. 1, pp. 340-345, 2001.

[3] P. Hämäläinen, J. H. Meurman, M. Keskinen, and E. Heikkinen, "Relationship between dental health and 10-year mortality in a cohort of community-dwelling elderly people," European Journal of Oral Sciences, vol. 111, no. 4, pp. 291-296, 2003.

[4] C. Cabrera, M. Hakeberg, M. Ahlqwist et al., "Can the relation between tooth loss and chronic disease be explained by socioeconomic status? A 24-year follow-up from the Population Study of Women in Gothenburg, Sweden," European Journal of Epidemiology, vol. 20, no. 3, pp. 229-236, 2005.

[5] N. Nakanishi, H. Fukuda, T. Takatorige, and K. Tatara, "Relationship between self-assessed masticatory disability and 9-year mortality in a cohort of community-residing elderly people," Journal of the American Geriatrics Society, vol. 53, no. 1 , pp. 54-58, 2005.

[6] C. C. Abnet, Y.-L. Qiao, S. M. Dawsey, Z.-W. Dong, P. R. Taylor, and S. D. Mark, "Tooth loss is associated with increased risk of total death and death from upper gastrointestinal 
cancer, heart disease, and stroke in a Chinese populationbased cohort," International Journal of Epidemiology, vol. 34, no. 2, pp. 467-474, 2005.

[7] P. Hämäläinen, J. H. Meurman, M. Kauppinen, and M. Keskinen, "Oral infections as predictors of mortality," Gerodontology, vol. 22, no. 3, pp. 151-157, 2005.

[8] M. Yoshida, H. Morikawa, M. Yoshikawa, K. Tsuga, and Y. Akagawa, "Eight-year mortality associated with dental occlusion and denture use in community-dwelling elderly persons," Gerodontology, vol. 22, no. 4, pp. 234-237, 2005.

[9] I. Morita, H. Nakagaki, K. Kato et al., "Relationship between survival rates and numbers of natural teeth in an elderly Japanese population," Gerodontology, vol. 23, no. 4, pp. 214$218,2006$.

[10] T. Österberg, G. E. Carlsson, V. Sundh, and B. Steen, "Number of teeth-a predictor of mortality in the elderly? A population study in three Nordic localities," Acta Odontologica Scandinavica, vol. 65, no. 6, pp. 335-340, 2007.

[11] T. Österberg, G. E. Carlsson, V. Sundh, and D. Mellström, "Number of teeth-a predictor of mortality in 70-year-old subjects," Community Dentistry and Oral Epidemiology, vol. 36, no. 3, pp. 258-268, 2008.

[12] J. H. Meurman and P. Hämäläinen, "Oral health and morbidity-implications of oral infections on the elderly," Gerodontology, vol. 23, no. 1, pp. 3-16, 2006.

[13] S. Ajwani, K. J. Mattila, R. S. Tilvis, and A. Ainamo, "Periodontal disease and mortality in an aged population," Special Care in Dentistry, vol. 23, no. 4, pp. 125-130, 2003.

[14] D. Jackson and J. Murray, "The loss of teeth in dentate populations," The Dental Practitioner and Dental Record, vol. 22, no. 5, pp. 186-189, 1972.

[15] J. D. Manson, "The elderly dental cripple," Proceedings of the Royal Society of Medicine, vol. 66, no. 6, pp. 597-598, 1973.

[16] A. Paganini-Hill and G. Hsu, "Smoking and mortality among residents of a California retirement community," American Journal of Public Health, vol. 84, no. 6, pp. 992-995, 1994.

[17] A. Paganini-Hill, C. H. Kawas, and M. M. Corrada, “Type of alcohol consumed, changes in intake over time and mortality: The Leisure World Cohort Study," Age and Ageing, vol. 36, no. 2, pp. 203-209, 2007.

[18] A. Paganini-Hill, C. H. Kawas, and M. M. Corrada, "Nonalcoholic beverage and caffeine consumption and mortality: The Leisure World Cohort Study," Preventive Medicine, vol. 44, no. 4, pp. 305-310, 2007.

[19] A. Paganini-Hill, C. H. Kawas, and M. M. Corrada, "Activities and mortality in the elderly: The Leisure World Cohort Study," Journals of Gerontology Series A, vol. 66A, no. 5, pp. 559-567, 2011.

[20] M. M. Corrada, C. H. Kawas, F. Mozaffar, and A. PaganiniHill, "Association of body mass index and weight change with all-cause mortality in the elderly," American Journal of Epidemiology, vol. 163, no. 10, pp. 938-949, 2006.

[21] A. Sheiham, J. G. Steele, W. Marcenes, S. Finch, and A. W. G. Walls, "The relationship between oral health status and Body Mass Index among older people: a national survey of older people in Great Britain," British Dental Journal, vol. 192, no. 12, pp. 703-706, 2002.

[22] N. Shah, H. Parkash, and K. R. Sunderam, "Edentulousness, denture wear and denture needs of Indian elderly-a community-based study," Journal of Oral Rehabilitation, vol. 31, no. 5, pp. 467-476, 2004.

[23] T. Yanagisawa, M. Ueno, K. Shinada, S. Ohara, F. A. C. Wright, and Y. Kawaguchi, "Relationship of smoking and smoking cessation with oral health status in Japanese men," Journal of Periodontal Research, vol. 45, no. 2, pp. 277-283, 2010.

[24] T. Dietrich, N. N. Maserejian, K. J. Joshipura, E. A. Krall, and R. I. Garcia, "Tobacco use and incidence of tooth loss among US male health professionals," Journal of Dental Research, vol. 86, no. 4, pp. 373-377, 2007.

[25] L. B. Copeland, E. A. Krall, L. J. Brown, R. I. Garcia, and C. F. Streckfus, "Predictors of tooth loss in two US adult populations," Journal of Public Health Dentistry, vol. 64, no. 1, pp. 31-37, 2004.

[26] P. Norlén, I. Johansson, and D. Birkhed, "Impact of medical and life-style factors on number of teeth in 68-year-old men in southern Sweden," Acta Odontologica Scandinavica, vol. 54, no. 1, pp. 66-74, 1996.

[27] National Heart, Lung, and Blood Institute, "Clinical guidelines on the identification, evaluation, and treatment of overweight and obesity in adults: the evidence report," 1998, National Heart, Lung, and Blood Institute,Bethesda, Md, USA, http://www.nhlbi.nih.gov/guidelines/obesity/ob_gdlns.pdf.

[28] D. R. Cox, "Regression models and life tables (with discussion)," Journals of the Royal Statistical Society B, vol. 34, pp. 187-220, 1972.

[29] A. Paganini-Hill and R. K. Ross, "Reliability of recall of drug usage and other health-related information," American Journal of Epidemiology, vol. 116, no. 1, pp. 114-122, 1982.

[30] A. Paganini-Hill and A. Chao, "Accuracy of recall of hip fracture, heart attack, and cancer: a comparison of postal survey data and medical records," American Journal of Epidemiology, vol. 138, no. 2, pp. 101-106, 1993.

[31] A. Paganini-Hill, R. K. Ross, and B. E. Henderson, "Prevalence of chronic disease and health practices in a retirement community," Journal of Chronic Diseases, vol. 39, no. 9, pp. 699-707, 1986.

[32] T. Bogg and B. W. Roberts, "Conscientiousness and healthrelated behaviors: a meta-analysis of the leading behavioural contributtors to mortality," Pyscologial Bulletin, vol. 130, pp. 887-919, 2004.

[33] M. L. Kern and H. S. Friedman, "Do conscientious individuals live longer? A quantitative review," Health Psychology, vol. 27, no. 5, pp. 505-512, 2008.

[34] A. Choo, D. M. Delac, and L. B. Messer, "Oral hygiene measures and promotion: review and considerations," Australian Dental Journal, vol. 46, no. 3, pp. 166-173, 2001. 


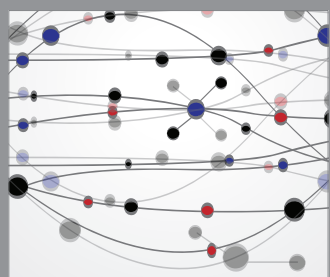

The Scientific World Journal
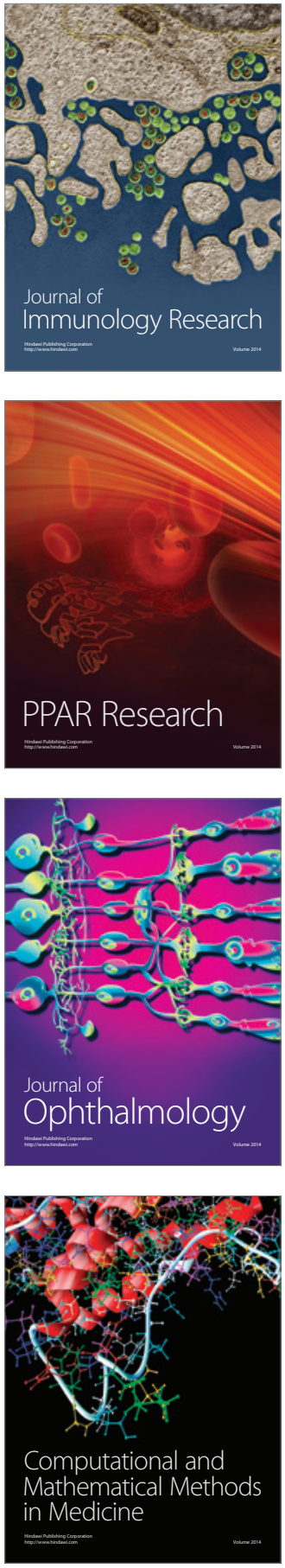

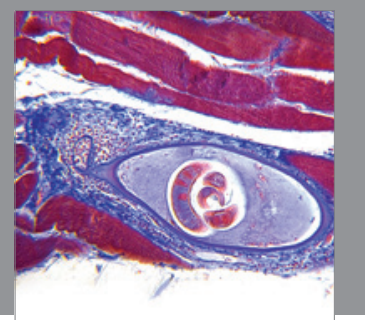

Gastroenterology

Research and Practice
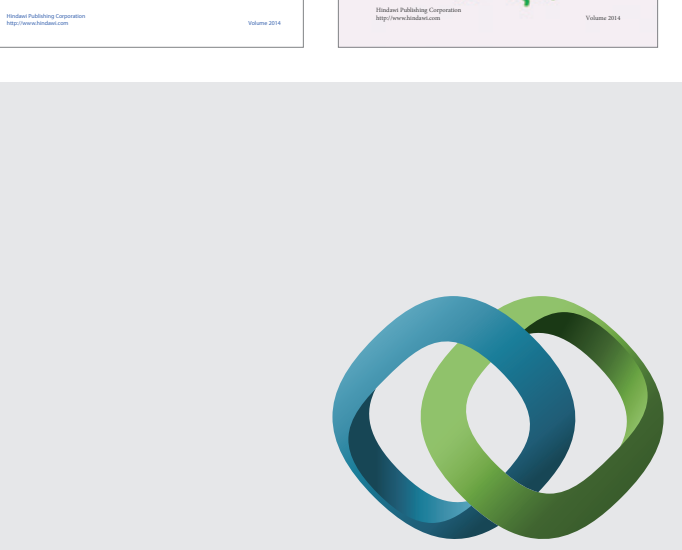

\section{Hindawi}

Submit your manuscripts at

http://www.hindawi.com
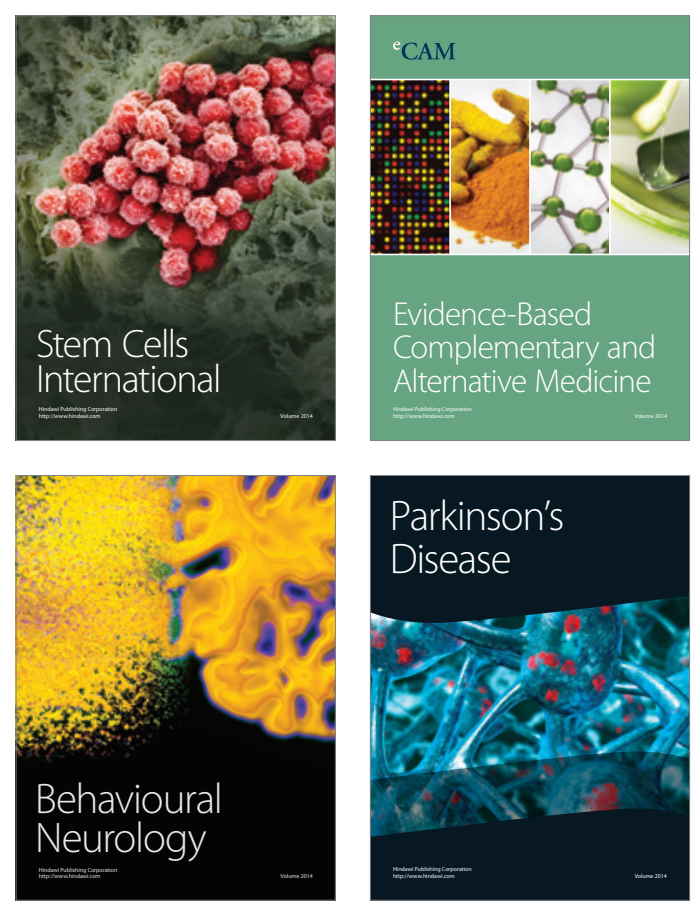

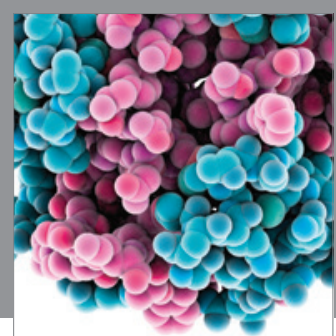

Journal of
Diabetes Research

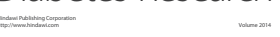

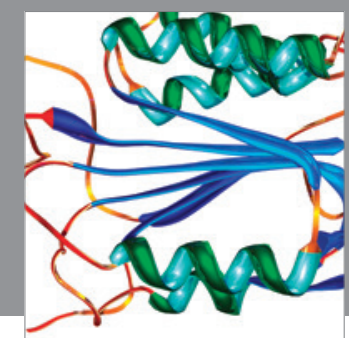

Disease Markers
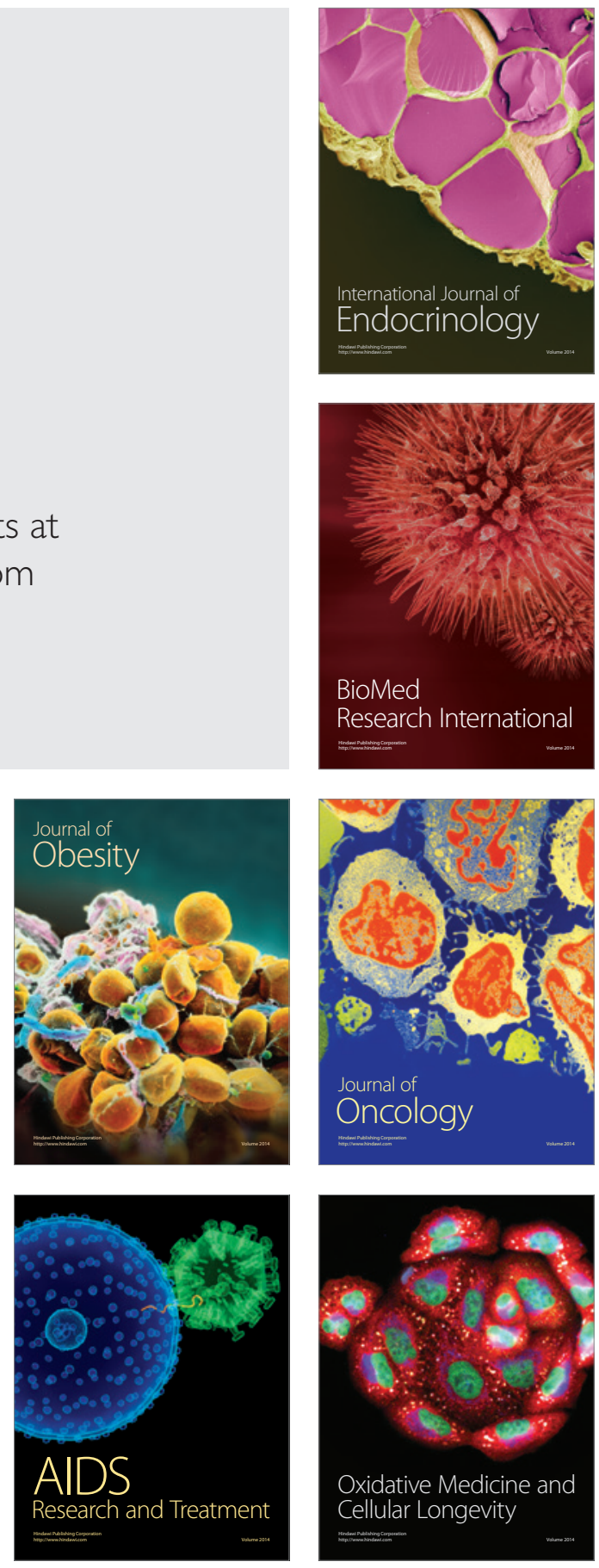